\title{
Cross-sectional and longitudinal associations between psychotic and depressive symptoms in depressed adolescents
}

\author{
Fiona Kehinde ${ }^{1,8} \cdot$ Aamena Valiji Bharmal $^{1,7} \cdot \operatorname{lan}$ M. Goodyer ${ }^{2} \cdot$ Raphael Kelvin $^{3} \cdot$ Bernadka Dubicka $^{6}$. \\ Nick Midgley ${ }^{4,5}$. Peter Fonagy ${ }^{4,5}$. Peter B. Jones ${ }^{2,3}$. Paul Wilkinson ${ }^{2,3}$ (1) on behalf of IMPACT Consortium
}

Received: 2 July 2020 / Accepted: 7 December 2020 / Published online: 11 January 2021

(c) The Author(s) 2021

\begin{abstract}
Adults with major depressive disorder (MDD) with psychotic features (delusions and/or hallucinations) have more severe symptoms and a worse prognosis. Subclinical psychotic symptoms are more common in adolescents than adults. However, the effects of psychotic symptoms on outcome of depressive symptoms have not been well studied in adolescents. Depressed adolescents aged 11-17 with and without psychotic symptoms were compared on depression severity scores at baseline and at 28- or 42-week follow-up in two large UK cohorts. Psychotic symptoms were weakly associated with more severe depression at baseline in both cohorts. At follow-up, baseline psychotic symptoms were only associated with depressive symptoms in one sample; in the other, the effect size was close to zero. This supports the DSM5 system of psychotic symptoms being a separate code to severity rather than the ICD10 system which only allows the diagnosis of psychotic depression with severe depression. There was no clear support for psychotic symptoms being a baseline marker of treatment response.
\end{abstract}

Keywords Psychotic symptoms $\cdot$ Adolescence $\cdot$ Unipolar depression $\cdot$ Depression severity

\section{Introduction}

Major depressive disorder is an important problem in adults and adolescents, with cumulative incidence in adolescence of $13 \%$ [1]. Adults with major depressive disorder (MDD) and psychotic features (delusions and/or hallucinations) have more severe symptoms [2,3], a worse prognosis [4, 5], greater risk of relapse [5, 6] and a higher mortality [7]. More broadly, a recent large study in the UK demonstrated

Fiona Kehinde and Aamena Valiji Bharmal are joint first authors.

Paul Wilkinson

pow12@cam.ac.uk

Fiona Kehinde

fiona.kehinde@hotmail.co.uk

Aamena Valiji Bharmal

aamena.bharmal@gmail.com

Ian M. Goodyer

ig104@cam.ac.uk

1 School of Clinical Medicine, University of Cambridge, Cambridge, UK

2 Department of Psychiatry, University of Cambridge, Douglas House, 18b Trumpington Road, Cambridge CB2 8AH, UK that psychotic experiences were associated with more severe depressive and anxiety symptoms and lower probability of recovery [8]. The role of psychotic symptoms in adolescent depression is less well studied. In the general population, there is a mean prevalence rate of $5 \%$ of subclinical psychotic symptoms [9]; compared to adults, these subclinical symptoms appear to be more common in children and adolescents, at around $7.5 \%$, and are often transitory [10,11]. Given these differences in prevalence of psychotic symptoms, it is possible that psychotic symptoms play a different role in adolescent compared with adult depression.

3 Cambridgeshire and Peterborough NHS Foundation Trust, Cambridge, UK

4 Anna Freud National Centre for Children and Families, London, UK

5 Research Department of Clinical, Educational and Health Psychology, University College London, London, UK

6 University of Manchester, Manchester, UK

7 Imperial GP VTS Scheme, London, UK

8 Royal Free Hospital, London, UK 
The two major international psychiatric classification systems view psychotic depression differently. ICD-10 assumes a unidimensional model, in which psychotic major depressive disorder can only be categorized under the 'severe' category [12]. This implies that psychotic symptoms cannot be found in mild/moderate depression. Contrastingly, the DSM5 has recently revised its classification: psychotic features can be coded alongside depression, but this is now separated from severity ratings [13]. It is unclear which viewpoint on psychotic depression is appropriate for depressed adolescents. This is important for clinicians, some of whom may assume that the presence of psychotic symptoms automatically means the depression is 'severe,' which may or may not be correct.

Psychotic depression at 12 years of age is associated with poorer educational, occupational and social outcomes by 16-20 years; however, it is not known whether this represents the likely outcome of psychotic symptoms across the adolescent years [14]. A recent symptom-level analysis of two adolescent to young adult ( $<26$ years old) community cohorts in the UK demonstrated that depressive and psychotic symptoms lie on the same continuum, with psychotic symptoms more common at the severe end [15]. This suggests that psychotic symptom emergence is an expression of clinical severity rather than a distinct category of affective disorder. If so then, as noted with adults, psychotic symptoms would be associated with poor treatment response.

We undertook secondary analyses using two cohorts recruited to randomized controlled trials of treatment for adolescent depression to compare the clinical features of depressed adolescents with and without psychotic symptoms at baseline and post-therapy follow-up. In particular, we looked for degree of correlation between severity of depressive symptoms and psychotic symptoms at baseline and follow-up. We tested whether psychotic symptoms at baseline:

a. Were associated with current severity of depressive symptoms.

b. Were associated with poor response to treatment.

\section{Methods}

\section{Participants}

Participants were recruited from two randomized controlled trials of depressed adolescents conducted in National Health Service specialist Child and Adolescent Mental Health clinics in the UK. As both trials showed no significant differences between treatment groups, participants from all treatment groups were combined within each dataset.
Adolescent depression antidepressants and psychotherapy trial (ADAPT)

ADAPT recruited 208 adolescents from the Cambridgeshire and Greater Manchester areas from 2000 to 2004 [16]. Inclusion criteria were: ages 11-17; current full or probable (at least four depressive symptoms) DSM-IV major depressive disorder; and significant social impairment. Individuals were excluded if they were not suitable to be in the treatment study: immediate admission required, significant learning disability, organic cause for depression, bipolar disorder, schizophrenia or selective serotonin reuptake inhibitors (SSRIs) contraindicated. Of note as per trial protocol in ADAPT (but not IMPACT), 34/249 (14\%) of young people initially suitable for the study were excluded because they improved following a brief initial intervention.

Participants were randomized to selective serotonin reuptake inhibitor (SSRIs) plus routine psychosocial care $(n=103)$ or selective serotonin reuptake inhibitor (SSRIs), routine psychosocial care plus cognitive-behavioural therapy (CBT, $n=105$ ). The routine psychosocial care delivered in both arms reflected NHS practice, including listening, support and problem solving. Treatment in both arms occurred throughout the 28 -week study period, gradually reducing in frequency. Mean (sd) number of sessions in the two treatment arms were, respectively, 6.5 (4.0) and 10.6 (5.7). Through the study period (28 weeks), there were no statistically significant differences between the treatment groups on any clinical outcome (all $p>0.3$ ) [17]

The study was approved and monitored by the North West Multi-Centre Research Ethics Committee and all local research ethics committees. Each participant and one adult with parental responsibility provided written informed consent.

\section{Improving mood with psychoanalytic and cognitive therapies (IMPACT)}

IMPACT recruited 465 adolescents from the Cambridgeshire, North London and Greater Manchester areas from 2010 to 2013 [18]. Adolescents aged 11-17 years with a DSMIV diagnosis of unipolar major depressive disorder were recruited. Exclusion criteria included a primary diagnosis of either bipolar disorder, schizophrenia or an eating disorder, significant learning disability or pervasive developmental disorder, pregnancy, substance abuse disorders, selective serotonin reuptake inhibitor (SSRI) use contraindicated or previous completion of one of the study treatments.

Participants were randomized to cognitive behaviour therapy (CBT, $n=154$ ), short-term psychoanalytic psychotherapy (STPP, $n=156$ ) or a brief psychosocial intervention (BPI $n=155$; this was a manualized form of the structured clinical care delivered to all participants in ADAPT). 
Median (IQR) number of sessions in each arm was less than planned: CBT 9 (5-14) over a mean (sd) of 25 (18) weeks; STPP 11 (5-23) over a mean (sd) of 28 (17) weeks; BPI 6 (4-11) over a mean (sd) of 28 (22) weeks. Participants in any arm were also permitted to receive SSRIs as part of their treatment in adherence with UK NICE guidelines for the treatment of unipolar depression (35\%).

Participants were assessed at baseline, 6, 12, 36, 52 and 86 weeks post-randomization by assessors masked to treatment allocation. To enable comparison with the ADAPT study, this analysis will use data from baseline and 36 weeks (end of treatment period). At 36 weeks, self-reported depression symptoms did not differ significantly between any of the three treatment groups (all $p>0.06$ ) [18].

The study was approved by the Cambridgeshire 2 Research Ethics Committee (reference 09/H0308/137) and local NHS provider trusts. All patients and their parents gave informed written consent.

\section{Instruments}

\section{Diagnosis: kiddie schedule for affective disorders and schizophrenia, present and lifetime version (K-SADS-PL)}

The K-SADS-PL was used to measure whether DSM-IV diagnoses (major depression plus psychiatric comorbid disorders) were present, with scores brought together to a best estimate by consensus rating. Both participant and a parent were interviewed separately [19]. The psychotic symptoms group was defined as having delusions and/or hallucinations currently present at threshold level on the K-SADSPL. Importantly, this measure was not able to distinguish whether psychotic symptoms were mood congruent/part of the depression itself. If data were missing on both items, or missing on one item and symptom absent on the other, the participant was excluded from analysis.

\section{Depressive symptoms}

The Mood and Feelings Questionnaire (MFQ) was used to measure participants' self-reported depressive symptoms in both trials. Participants rated a set of 33 items (covering the range of DSM-IV depressive symptoms) over the last 2 -week period [20]. The MFQ has good test-retest reliability, internal consistency and discriminant validity in clinical adolescent samples [21, 22].

\section{Sensitivity analyses}

Due to potential issues arising from self-rated questionnaires, analyses were repeated using the well-validated
Children's Depression Rating Scale-Revised, an observerrated measure of depressive symptoms (only available for ADAPT) [23]. To test associations between psychotic symptoms and social function, analyses were repeated the sum of the social function items (HoNOSCA-Fx) of the Health of the Nations Outcome Scales for Children and Adolescents [HoNOSCA, items 5 (scholastic/language skills), 10 (peer relationships), 11 (self-care and independence), 12 (family), 13 (school attendance)]. The HoNOSCA is an observer-rated measure of mental health status, with 13 items across a range of symptom areas, behaviours and social function [24].

\section{Statistical analysis}

Data analysis was performed using Stata version 14 (StataCorp, College Station, Tex.).

Only participants will full major depressive disorder and complete data for the psychosis variable at baseline were included. Depressive symptoms/functional baseline and follow-up were compared between those with and without baseline psychotic symptoms. Results were controlled for appropriate covariates, using structural equation modelling (ADAPT: region, age, gender; plus baseline value and treatment group at follow-up. IMPACT: treatment group, region, age, gender, ethnicity and SSRI treatment; plus baseline value, treatment group and time taken to start treatment at follow-up). To control for attrition, the full information maximum likelihood (FIML) option was performed.

\section{Results}

192/208 of participants from ADAPT had full major depressive disorder at baseline. 190/192 (99\%) from ADAPT and 439/465 (94\%) from IMPACT had data on baseline psychotic symptoms. In ADAPT, 18 (9.5\%) had threshold psychotic symptoms: 14 had hallucinations alone, and 4 had hallucinations plus delusions. In IMPACT, 42 (9.6\%) had threshold psychotic symptoms: 28 had hallucinations alone, 8 had delusions alone, and 6 had hallucinations plus delusions. Table 1 shows baseline characteristics of both studies. Gender balance and prevalence of psychotic symptoms were similar in both studies. Ethnic diversity, baseline age and depression severity (as measured by MFQ score) were significantly higher in IMPACT compared to ADAPT. Proportion of participants with comorbid psychiatric disorders and impairment of social function was significantly higher for ADAPT.

Univariate analysis (Table 2 and Fig. 1) demonstrated baseline self-rated depressive symptoms to be significantly 
Table 1 Baseline demographic and clinical variables for participants in the ADAPT and IMPACT studies

\begin{tabular}{llll}
\hline & ADAPT & IMPACT & Difference \\
\hline $\begin{array}{l}\text { Sample size } \\
\text { Gender }\end{array}$ & $n=190$ & $n=439$ & \\
$\quad$ Male & $47(25 \%)$ & $110(25 \%)$ & $X^{2}(d f 1)=0.01, p=0.9$ \\
Female & $143(75 \%)$ & $329(75 \%)$ & \\
Age, mean (sd) & $14.7(1.2)$ & $15.6(1.4)$ & $Z=7.2, p<0.00005$ \\
Baseline MFQ, mean (sd) & $39.3(11.6)$ & $45.4(10.5)$ & $Z=5.8, p<0.00005$ \\
Baseline HoNOSCA-Fx, mean (sd) & $10.6(3.5)$ & $6.7(3.5)$ & $Z=11.0, p<0.00005$ \\
Ethnicity* white & $173 / 179(97 \%)$ & $345 / 431(80 \%)$ & $X^{2}(d f 1)=27, p<0.0005$ \\
Number with at least one comorbid disorder & $169(89 \%)$ & $213 / 439(49 \%)$ & $X^{2}(d f 1)=91, p<0.0005$ \\
Number with psychotic symptoms & $18(9.5 \%)$ & $42(9.6 \%)$ & $X^{2}(d f 1)=0.0, p=1.0$ \\
\hline
\end{tabular}

$M F Q$ Mood and Feelings Questionnaire. HoNOSCA-Fx Social function items $(5,10,11,12,13)$ of the Health of the Nation Outcome Scales for Children and Adolescents

Table 2 Associations between baseline psychotic symptoms and baseline and post-treatment depressive symptoms/social function

\begin{tabular}{|c|c|c|c|c|c|c|c|}
\hline Clinical measures & $N$ for univariate & $\begin{array}{l}\text { No. psychotic } \\
\text { symptoms mean } \\
\text { (sd) }\end{array}$ & $\begin{array}{l}\text { Psychotic dymp- } \\
\text { toms present mean } \\
(\mathrm{sd})\end{array}$ & $\begin{array}{l}\text { Univariate } \\
\text { comparison }(t \text { or } \\
\text { Mann-Whitney } Z \text { ) }\end{array}$ & Univariate $p$ & Multivariate beta & $\begin{array}{l}\text { Multivariate } \\
p \text { (FIML) }\end{array}$ \\
\hline \multicolumn{8}{|l|}{ ADAPT } \\
\hline \multicolumn{8}{|l|}{ MFQ } \\
\hline Baseline & 190 & $38.8(11.4)$ & $44.1(12.8)$ & $Z=-2.08$ & 0.04 & 0.122 & 0.087 \\
\hline 28 weeks & 173 & $16.3(14.7)$ & $28.3(19.2)$ & $Z=-2.45$ & 0.01 & 0.171 & 0.010 \\
\hline \multicolumn{8}{|l|}{ CDRS-R } \\
\hline Baseline & 190 & $59.2(10.1)$ & $64.4(7.4)$ & $Z=-2.21$ & $\mathbf{0 . 0 3}$ & 0.174 & 0.013 \\
\hline 28 weeks & 175 & $34.5(14.1)$ & $44.3(17.4)$ & $Z=-2.35$ & 0.02 & 0.142 & 0.028 \\
\hline \multicolumn{8}{|l|}{ HoNOSCA-Fxn } \\
\hline Baseline & 190 & $10.4(3.7)$ & $12.2(3.3)$ & $t(d f 188)=2.05$ & 0.042 & 0.157 & 0.027 \\
\hline 28 weeks & 176 & $6.9(5.3)$ & $8.0(4.9)$ & $t(d f 174)=1.1$ & 0.3 & 0.055 & 0.4 \\
\hline \multicolumn{8}{|l|}{ IMPACT } \\
\hline \multicolumn{8}{|l|}{ MFQ } \\
\hline Baseline & 439 & $45.2(10.5)$ & $51.3(9.7)$ & $Z=-3.72$ & 0.0002 & 0.143 & 0.002 \\
\hline 42 weeks & 299 & $26.5(15.7)$ & $29.8(18.1)$ & $Z=-0.82$ & 0.4 & -0.003 & 0.96 \\
\hline \multicolumn{8}{|l|}{ HoNOSCA-Fxn } \\
\hline Baseline & 396 & $6.8(3.5)$ & $6.4(4.0)$ & $Z=0.5$ & 0.6 & -0.035 & 0.5 \\
\hline 42 weeks & 226 & $3.4(3.3)$ & $3.5(3.3)$ & $Z=0.2$ & 0.8 & -0.006 & 0.9 \\
\hline
\end{tabular}

Bold value indicates $p<0.05$

$M F Q$ Mood and Feelings Questionnaire, CDRS-R Children's Depression Rating Scale—Revised, HoNOSCA-Fx Social function items (5, 10, 11, 12, 13) of the Health of the Nation Outcome Scales for Children and Adolescents

higher in participants with psychotic symptoms in both studies (all $p<0.05)$. When controlling for relevant covariates (Table 2), this difference continued to be statistically significant in IMPACT $(\beta=0.143, p=0.002)$ but not in ADAPT $(\beta=0.122, p=0.087)$.

Time from baseline to post-treatment assessments [median (IQR)] for ADAPT was 28.3 (1.4) weeks and for IMPACT was 41.7 (4.1) weeks. In ADAPT and IMPACT respectively, post-treatment MFQ was complete for 173/190 $(91 \%)$ and 299/439 (68\%) participants included in the analysis. Univariate analysis demonstrated that participants with baseline psychotic symptoms had greater severity of depression post-treatment in ADAPT $(Z=2.45 ; p=0.01)$ but not in IMPACT ( $Z=0.8 ; p=0.4$, Table 2$)$. After controlling for relevant covariates in FIML analysis (including baseline severity of depression), baseline psychotic symptoms were associated with higher post-treatment depressive symptoms in ADAPT $(\beta=0.171, p=0.010$, Table 2$)$ but not in IMPACT $(\beta=-0.003, p=0.96$, Table 2$)$. This analysis was repeated for IMPACT at the previous assessment point (median (IQR) 17.1 (4.1) weeks). Baseline psychotic symptoms continued 

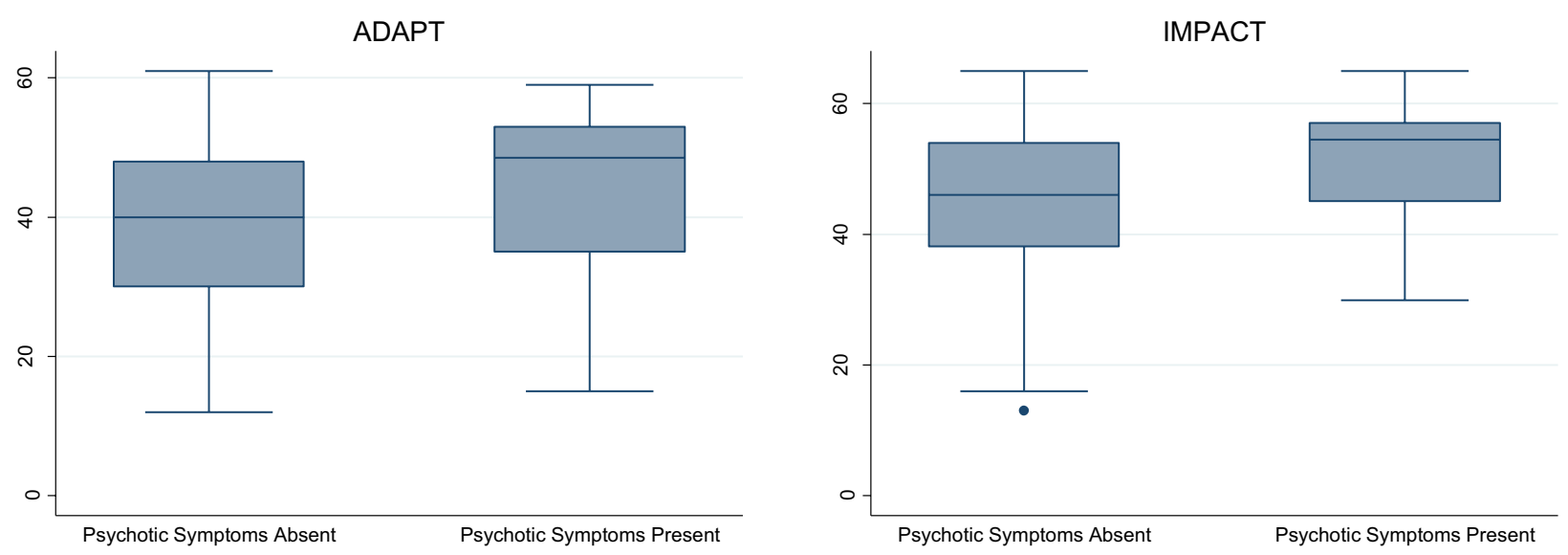

Key: MFQ: Mood and Feelings Questionnaire

Fig. 1 Baseline depressive symptoms in adolescents with and without psychotic symptoms

not to be associated with follow-up depressive symptoms $(\beta=-0.004, p=0.9)$.

Complete case analysis at follow-up revealed similar multivariate results: Baseline psychotic symptoms were associated with higher depressive symptoms in ADAPT $(n=173$, $\beta=0.178, p=0.010)$ but not IMPACT $(n=291, \beta=0.007$, $p=0.91)$. Results were similar for observer-rated depressive symptoms (CDRS-R) as self-rated symptoms (ADAPT only; baseline: $\beta=0.174, p=0.013$; follow-up: $\beta=0.142$, $p=0.028$ ). However for function (HoNOSCA-Fx), psychotic symptoms were associated with worse function at baseline in ADAPT $(\beta=0.157, p=0.027)$ but not in IMPACT $(\beta=-0.035, p=0.5)$; in both samples, baseline psychotic symptoms were not significantly associated with follow-up function $(p>0.4)$ (Table 2).

\section{Discussion}

In this study, we wanted to explore the hypotheses that, in depressed adolescents without a primary diagnosis of bipolar disorder or schizophrenia, psychotic symptoms at baseline are: (a) strongly associated with severity of disorder and (b) a marker for poor treatment response. We used data from two independent cohorts, to allow replication.

In both cohorts, baseline depressive symptoms were higher in adolescents with psychotic symptoms. This remained significant when controlling for important confounders in the larger IMPACT sample, but was of borderline significance in ADAPT. Similar beta coefficients suggest that this may have been a type II error in ADAPT, especially as sensitivity analyses using the CDRS-R were significant. At first sight, this may seem to support the ICD10 viewpoint that psychotic symptoms are only a feature of severe depression. However, and as can be seen clearly in Fig. 1, there was still great overlap between those with and without psychotic symptoms, and an adjusted standardized beta around 0.14 makes the effect size smaller than the standard 0.2 threshold for 'small' effects. The presence of significant numbers of young people with psychotic symptoms but depression at the milder end, and significant numbers without psychotic symptoms but severe depression supports the more flexible DSM5 viewpoint that psychotic symptoms can be coded at all levels of depression severity. Therefore, clinicians should not assume that psychotic symptoms mean that depression must be severe-instead they should measure severity directly. These findings are in keeping with recent evidence that psychotic experiences are seen across multiple mental disorders across the full spectrum of severity [25].

Findings on the prognostic implications of psychotic symptoms were mixed. In the ADAPT sample, psychotic symptoms were indeed associated with greater severity of depressive symptoms post-treatment, even when controlling for confounders including baseline severity. However, this finding was not replicated in IMPACT, and the very low standardized beta coefficient (of the opposite sign to expected: - 0.003) makes this very unlikely to be a type II error.

There are several possible explanations for the significant findings in one sample but not the other. The first is different sample characteristics, with there being a possibility of different predictive effects of psychotic symptoms within different 'subtypes' of depression. There were some significant differences between samples, although these operated in opposite directions, with greater severity of depressive symptoms in IMPACT, but higher levels of comorbidity and reduced social function in ADAPT. Also ADAPT 
had a higher proportion of white British participants and excluded the $14 \%$ of participants who responded to a brief intervention. And while all participants in ADAPT received SSRI antidepressants during the study, only about $30 \%$ of the IMPACT sample did. However, all participants received a psychological intervention in both studies: CBT, STPP or a Brief Psychosocial Intervention. Importantly, modality of psychological therapy had no effect on outcome. Importantly, prevalence of psychotic symptoms was the same in both samples (around 10\%), and the zero effect size of psychotic symptoms on outcome in IMPACT makes it unlikely that a subgroup matching ADAPT would have a significant positive result. Second, the samples were recruited around ten years apart. Services may have had different referral thresholds. Differences in population behaviour may have led to differences, for example smoking of greater and more potent cannabis by British adolescents in recent years [26], which may make lead to a different 'type' of psychotic symptoms in depression. Although if this were the case in this sample, this would be likely to make psychosis rates different and, again, would be unlikely to account for an effect size of virtually zero. Third, post-treatment assessments occurred at different times after baseline (28 vs 42 weeks), and regression to the mean may have meant depressive symptoms no longer differed in our groups in the final IMPACT assessments; we therefore also compared groups at 17 weeks and again found no effect of baseline psychotic symptoms, making this explanation unlikely. The most likely explanation is that the finding in ADAPT was a chance observation, given non-replication in an independent (and much larger) sample, and lack of significant effects of baseline psychotic symptoms on social function at followup. Indeed, much has been written about the problem of 'significant' findings being published when they are in fact false; and replication is well recognized as being crucial to give us confidence that findings are in fact real [27]. This conclusion that results from the smaller ADAPT study could be a false positive is further supported by results from a recent meta-analysis of depressed adults, which found that the difference between post-follow-up depression severity in those with and without psychotic features was nonsignificant in studies with larger sample sizes ( $n$ cases $>50)$ [5]. It is also possible that differences in prognostic effects between adults and adolescents may be because of differences in the nature, aetiology and treatment responsivity of adolescent depression (in particular the fact that adolescent depression is usually first episode) [28], and differences in the nature of psychotic symptoms in adults and adolescents [10].

The contrasting results do not make it clear how depressed adolescents with psychotic symptoms should be treated-in IMPACT, the same treatments are as effective in reducing depressive symptoms in depression with and without psychotic features. However, this was not the case in ADAPT.
There were however large reductions in depressive symptoms in both groups in both studies, so it is reasonable to use the same treatments as used in these RCTs to treat adolescents. Further larger studies of adolescents with depression with psychotic symptoms are needed to determine whether there is a need for additional treatment for such patients.

\section{Limitations}

Both the ADAPT and IMPACT studies were initially designed as treatment comparison studies, and there were no primary hypotheses about the effects of psychotic symptoms. There is therefore a risk of type I errors in this secondary analysis. Crucially, psychotic symptoms were only asked about at as a current yes/no single question as part of the K-SADS-PL. This does not give any detail about the nature of the psychotic symptoms nor the time course (in particular whether the psychotic symptoms predated the depression). An important limitation of this is that our study could not determine whether these psychotic symptoms were specifically part of psychotic depression. While adolescents with bipolar disorder and schizophrenia were excluded from the study, it is possible that some adolescents had psychotic symptoms that predated the depression and may or may not have been part of a mental disorder. Our study can therefore only answer the question (itself useful) or what the implications of non-specific psychotic symptoms are on depressive symptoms. Furthermore, a continuous measure of psychotic symptoms would likely have had greater power than a simple yes/no question.

In IMPACT, a self-rated questionnaire of depressive symptoms only was used; however observer-rated and selfrated questionnaires led to similar results in ADAPT, and so this is unlikely to account for inaccuracies/differences in results. In addition, other potentially interesting explanatory variables were not enquired about, including childhood maltreatment, which is associated with psychotic symptoms, more severe depressive symptoms and poor prognosis [29]. While bipolar disorder was an exclusion criterion for both studies, some participants may have been in the early stages of bipolar disorder, with no manic episodes yet having occurred; again bipolar disorder is associated with psychotic symptoms, greater severity and poor prognosis; and bipolar disorder with psychosis is associated with a particularly poor prognosis $[30,31]$. We also did not have information on lifetime course of depression, in particular presence of prior episodes, and effects may have differed in recurrent vs first episode depression. Neither study used a valid comprehensive measure of social function; therefore, we needed to use a non-validated short subscale; a more comprehensive validated measure may have provided significant results.

The psychotic symptom group sample sizes are also small in both ADAPT and IMPACT $(<10 \%$ of the sample 
size in both cases); this reduces the statistical power and the robustness of the results from the statistical analysis. The two cohorts did have different findings; while it is more appropriate to make the conservative null conclusion we have done, this may be incorrect and result need replication in a third sample. In addition, participants in both studies, as with all RCTs, were not representative of the community population (in particular because they were seeking help and agreed to being in a treatment study) — therefore, results may not generalize to depressed adolescents in general.

To answer these questions authoritatively, a study needs to collect a larger proportion of adolescents with psychotic symptoms and measure psychotic symptoms using detailed and well-validated scales, with details on longitudinal pattern of depressive episodes and psychotic symptoms.

\section{Clinical implications}

There is a large overlap in severity between depressed adolescents with and without psychotic symptoms, and clinicians should not assume that psychotic symptoms necessarily mean severe depression and worse outcome. It is reasonable to continue to use evidence-based psychological therapies with or without an SSRI to treat adolescents with depression.

Author contributions $\mathrm{FK}, \mathrm{AB}$ and $\mathrm{PW}$, and IG were involved in the conception, design and analysis. All authors were involved in drafting, writing and final approval of the article. All authors agree to be accountable for all aspects of the accuracy and integrity of the work.

Funding Both ADAPT and IMPACT were funded by the National Institute for Health Research (NIHR) Health Technology Assessment (HTA) programme (Grant no. 06/05/01), and the Department of Health.

Availability of data and materials The data that support the findings of this study are available from the corresponding author PW, upon reasonable request.

Code availability The code is available from the corresponding author by request.

\section{Compliance with ethical standards}

Conflict of interest The authors declare that they have no conflict of interest.

Open Access This article is licensed under a Creative Commons Attribution 4.0 International License, which permits use, sharing, adaptation, distribution and reproduction in any medium or format, as long as you give appropriate credit to the original author(s) and the source, provide a link to the Creative Commons licence, and indicate if changes were made. The images or other third party material in this article are included in the article's Creative Commons licence, unless indicated otherwise in a credit line to the material. If material is not included in the article's Creative Commons licence and your intended use is not permitted by statutory regulation or exceeds the permitted use, you will need to obtain permission directly from the copyright holder. To view a copy of this licence, visit http://creativecommons.org/licenses/by/4.0/.

\section{References}

1. Kessler RC, Petukhova M, Sampson NA, Zaslavsky AM, Wittchen H-U (2012) Twelve-month and lifetime prevalence and lifetime morbid risk of anxiety and mood disorders in the United States. Int J Methods Psychiatric Res 21(3):169-184. https://doi. org/10.1002/mpr.1359

2. Østergaard SD, Bille J, Søltoft-Jensen H, Lauge N, Bech P (2012) The validity of the severity-psychosis hypothesis in depression. J Affect Disord 140(1):48-56. https://doi.org/10.1016/j. jad.2012.01.039

3. Forty L, Jones L, Jones I, Cooper C, Russell E, Farmer A, Craddock $\mathrm{N}$ et al (2009) Is depression severity the sole cause of psychotic symptoms during an episode of unipolar major depression? A study both between and within subjects. J Affect Disord 114(1-3):103-109. https://doi.org/10.1016/j.jad.2008.06.012

4. Hoch A, MacCurdy JT (1922) The prognosis of involution melancholia. Arch Neurol Psychiatry 7(1):1. https://doi.org/10.1001/ archneurpsyc.1922.02190070004001

5. Jääskeläinen E, Juola T, Korpela H, Lehtiniemi H, Nietola M, Korkeila J (2018) Epidemiology of psychotic depression-systematic review and meta-analysis. Psychol Med. https://doi. org/10.1017/S0033291717002501

6. Johnson J, Horwath E, Weissman MM (1991) The validity of major depression with psychotic features based on a community study. Arch Gen Psychiatry. https://doi.org/10.1001/archp syc.1991.01810360039006

7. Vythilingam M, Chen J, Bremner JD, Mazure CM, Maciejewski PK, Nelson JC (2003) Psychotic depression and mortality. Am J Psychiatry 160(3):574-576. https://doi.org/10.1176/appi. ajp.160.3.574

8. Knight C, Russo D, Stochl J, Croudace T, Fowler D, Grey N, Perez J et al (2020) Prevalence of and recovery from common mental disorder including psychotic experiences in the UK Primary Care Improving Access to Psychological Therapies (IAPT) Programme. J Affect Disord 272:84-90. https://doi.org/10.1016/j. jad.2020.04.015

9. Linscott RJ, van Os J (2010) Systematic reviews of categorical versus continuum models in psychosis: evidence for discontinuous subpopulations underlying a psychometric continuum. Implications for DSM-V, DSM-VI, and DSM-VII. Ann Rev Clin Psychol 6(1):391-419. https://doi.org/10.1146/annurev.clinpsy.03240 8.153506

10. van Os J, Linscott RJ, Myin-Germeys I, Delespaul P, Krabbendam L (2009) A systematic review and meta-analysis of the psychosis continuum: evidence for a psychosis proneness-persistenceimpairment model of psychotic disorder. Psychol Med 39(02):179. https://doi.org/10.1017/S0033291708003814

11. Kelleher I, Connor D, Clarke MC, Devlin N, Harley M, Cannon M (2012) Prevalence of psychotic symptoms in childhood and adolescence: a systematic review and meta-analysis of population-based studies. Psychol Med 42(09):1857-1863. https://doi. org/10.1017/S0033291711002960

12. World Health Organization. (1992). The ICD-10 classification of mental and behavioural disorders: Clinical descriptions and diagnostic guidelines. Geneva:World Health Organization

13. American Psychiatric Association (2013) Diagnostic and Statistical Manual of Mental Disorders: Diagnostic and Statistical Manual of Mental Disorders, 5th edn. Arlington, VA: American Psychiatric Association 
14. Davies J, Sullivan S, Zammit S (2018) Adverse life outcomes associated with adolescent psychotic experiences and depressive symptoms. Soc Psychiatry Psychiatr Epidemiol 53(5):497-507. https://doi.org/10.1007/s00127-018-1496-Z

15. Stochl J, Khandaker GM, Lewis G, Perez J, Goodyer IM, Zammit S, Jones PB et al (2015) Mood, anxiety and psychotic phenomena measure a common psychopathological factor. Psychol Med 45(7):1483-1493. https://doi.org/10.1017/S003329171400261X

16. Goodyer I, Dubicka B, Wilkinson P, Kelvin R, Roberts C, Byford $S$, Harrington R et al (2007) Selective serotonin reuptake inhibitors (SSRIs) and routine specialist care with and without cognitive behaviour therapy in adolescents with major depression: randomised controlled trial. BMJ 335:142. https://doi.org/10.1136/ bmj.39224.494340.55

17. Goodyer IM, Reynolds S, Barrett B, Byford S, Dubicka B, Hill J, Fonagy P et al (2017) Cognitive-behavioural therapy and short-term psychoanalytic psychotherapy versus brief psychosocial intervention in adolescents with unipolar major depression (IMPACT): a multicentre, pragmatic, observer-blind, randomised controlled trial. Health Technol Assess. https://doi.org/10.3310/ hta21120

18. Goodyer IM, Reynolds S, Barrett B, Byford S, Dubicka B, Hill J, Kelvin R et al (2017) Cognitive behavioural therapy and shortterm psychoanalytical psychotherapy versus a brief psychosocial intervention in adolescents with unipolar majordepressive disorder (IMPACT): a multicentre, pragmatic, observer-blind, randomised controlled superiority trial. Lancet Psychiatry 4(2):109-119. https ://doi.org/10.1016/S2215-0366(16)30378-9

19. Kaufman J, Birmaher B, Brent D, Rao U, Flynn C, Moreci P, Ryan $\mathrm{N}$ et al (1997) Schedule for affective disorders and schizophrenia for school-age children-present and lifetime version (K-SADSPL): initial reliability and validity data. J Am Acad Child Adolesc Psychiatry 36(7):980-988. https://doi.org/10.1097/00004 583-199707000-00021

20. Angold A, Weissman MM, John K, Merikangas KR, Prusoff BA, Wickramaratne P, Warner V et al (1987) Parent and child reports of depressive symptoms in children at low and high risk of depression. J Child Psychol Psychiatry 28(6):901-915

21. Kent L, Vostanis P, Feehan C (1997) Detection of major and minor depression in children and adolescents: evaluation of the mood and feelings questionnaire. J Child Psychol Psychiatry 38(5):565573. https://doi.org/10.1111/j.1469-7610.1997.tb01543.x

22. Wood A, Kroll L, Moore A, Harrington R (1995) Properties of the mood and feelings questionnaire in adolescent psychiatric outpatients: a research note. J Child Psychol Psychiatry 36(2):327-334. https://doi.org/10.1111/j.1469-7610.1995.tb01828.x

23. Poznanski EO, Grossman JA, Buchsbaum Y, Banegas M, Freeman L, Gibbons R (1984) Preliminary studies of the reliability and validity of the children's depression rating scale. J Am Acad Child Adolesc Psychiatry 23(2):191-197. http://www.ncbi.nlm.nih. gov/entrez/query.fcgi? $\mathrm{cmd}=$ Retrieve $\& \mathrm{db}=$ PubMed $\& d o p t=$ Citat ion\&list_uids $=6715741$

24. Gowers SG, Harrington RC, Whitton A, Lelliot P, Beevor A, Wing J, Jezzard R (1999) Health of the nation outcome scales for children and adolescents (HoNOSCA). Br J Psychiatry 174(5):428431. https://doi.org/10.1192/bjp.174.5.428

25. Perez J, Jones PB (2019) Breaking the web: life beyond the at-risk mental state for psychosis. Psychol Med. https://doi.org/10.1017/ S0033291719002605

26. Di Forti M, Morgan C, Dazzan P, Pariante C, Mondelli V, Marques TR, Murray RM et al (2009) High-potency cannabis and the risk of psychosis. Br J Psychiatry 195(6):488-491. https ://doi.org/10.1192/bjp.bp.109.064220

27. Ioannidis JPA (2005) Why most published research findings are false. PLoS Med 2(8):e124. https://doi.org/10.1371/journ al.pmed.0020124

28. Rice F, Riglin L, Lomax T, Souter E, Potter R, Smith DJ, Thapar A et al (2019) Adolescent and adult differences in major depression symptom profiles. J Affect Disord 243:175-181. https://doi. org/10.1016/j.jad.2018.09.015

29. Carson GA, Pataki C (2020) Affective disorders with psychosis in youth: an update. Child Adolesc Psychiatr Clin N Am 29:91-102

30. Østergaard SD, Straszek S, Petrides G, Skadhede S, Jensen SOW, Munk-Jørgensen P, Nielsen J (2014) Risk factors for conversion from unipolar psychotic depression to bipolar disorder. Bipolar Disord 16(2):180-189. https://doi.org/10.1111/bdi.12152

31. Shalev A, Merranko J, Gill MK, Goldstein T, Liao F, Goldstein BI, Birmaher B et al (2020) Longitudinal course and risk factors associated with psychosis in bipolar youths. Bipolar Disord 22(2):139-154. https://doi.org/10.1111/bdi.12877 